Psychotherapeut 2020 $65: 167-175$ https://doi.org/10.1007/s00278-020-00421-1 Online publiziert: 27. April 2020

(c) Der/die Autor(en) 2020

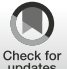

Moritz Bruno Petzold ${ }^{*}$ Felicitas Ernst ${ }^{1} \cdot$ Ursula Spitzer $^{2} \cdot$ Julian Gabrysch ${ }^{1}$. Nina Schweinfurth ${ }^{2} \cdot$ Norman Satorius $^{3}$. Andreas Ströhle ${ }^{1} \cdot$ Felix Betzler $^{1}$

${ }^{1}$ Klinik für Psychiatrie und Psychotherapie, Charité - Universitätsmedizin Berlin, Campus Mitte, corporate member of Freie Universität Berlin, Humboldt-Universität zu Berlin, and Berlin Institute of Health, Berlin, Deutschland

${ }^{2}$ Universitäre Psychiatrische Kliniken Basel, Basel, Schweiz

${ }^{3}$ Association for the Improvement of Mental Health Programmes (AMH), Genf, Schweiz

\title{
Ärztliche Vorbildfunktion bezüglich körperlicher Aktivität für Patienten mit psychischen Erkrankungen
}

\author{
Empfehlungsverhalten und \\ Selbstkonkordanz von Psychiatern und \\ ärztlichen Psychotherapeuten
}

\begin{abstract}
Körperliche Aktivität spielt eine bedeutende Rolle bei der Prävention und Therapie psychischer Erkrankungen. Zahlreiche Untersuchungen zeigen, dass ein großer Teil der Menschen mit psychischen Erkrankungen die Empfehlungen bezüglich körperlicher Aktivität nicht erfüllt. Psychiater/ärztliche Psychotherapeuten spielen eine Schlüsselrolle dabei, ihre Patienten über positive Auswirkungen körperlicher Aktivität aufzuklären und körperliche Aktivität zu empfehlen. Diese Studie untersucht das Empfehlungsverhalten von Psychiatern/ ärztlicher Psychotherapeuten sowie Einflussfaktoren darauf.
\end{abstract}

\section{Hintergrund}

Körperliche Aktivität kann eine wichtige Rolle in der Prävention und Thera-

Die Autoren Andreas Ströhle und Felix Betzler haben zu gleichen Teilen zum Manuskript beigetragen.

Aus Gründen derbesseren Lesbarkeit wirdim Beitrag das generische Maskulinum verwendet. Weibliche und anderweitige Geschlechteridentitäten sind ausdrücklich mitgemeint. pie von psychischen Erkrankungen spielen (Ströhle 2019). Menschen, die regelmäßig körperlich aktiv sind, zeigen geringere Inzidenzen bezüglich zahlreicher psychischer Erkrankungen (Ströhle et al. 2007); insbesondere für die Depression wurde in einer neuen Metaanalyse robuste Evidenz nachgewiesen (Schuch et al.2018). Neben der Prävention ist körperliche Aktivität auch bei der Behandlung psychischer Erkrankungen, wie Depression, Angststörungen und Schizophrenie, wirksam (Ashdown-Franks et al. 2019; Zschucke et al. 2013; Petzold et al. in press). Die Weltgesundheitsorganisation (WHO) empfiehlt als gesundheitsförderliches Ausmaß körperlicher Aktivität für Erwachsene mindestens $150 \mathrm{~min}$ moderate oder 75 min intensive körperliche Aktivität in Einheiten von jeweils 10 min sowie zusätzliches Krafttraining an mindestens 2 Tagen. Trotz der zahlreichen positiven Effekte erfüllt nur etwa ein Drittel der Menschen mit psychischen Erkrankungen die Empfehlungen bezüglich gesundheitsförderlicher Aktivität, zudem belegen bisherige Studien mit psychischen erkrankten Menschen ein geringeres Ausmaß körperlicher Aktivität als in der Allgemeinbevölkerung
(Kruisdijk et al. 2017; Petzold et al. 2017; Schuch et al. 2017).

Wie Menschen mit psychischen Erkrankungen darin unterstützt werden können, ihr Aktivitätsniveau zu steigern, stellt daher eine der wichtigsten Forschungsfragen im Bereich körperlicher Aktivität und psychischer Gesundheit dar (Vancampfort et al. 2016). Ein Großteil der existierenden Studien konzentriert sich auf Einzel- oder Gruppeninterventionen (Czosnek et al. 2018). Neben spezifischeren Interventionen spielt die allgemeine Beratung hinsichtlich körperlicher Aktivität durch die behandelnden Gesundheitsfachkräfte eine wichtige Rolle bezüglich der Förderung körperlicher Aktivität bei Menschen mit psychischen Erkrankungen. Bei der Förderung bzw. Beratung können Psychiater/ärztliche Psychotherapeuten auf wissenschaftlich fundierte Empfehlungen zurückgreifen (z. B. Haseler et al. 2019; American College of Sports Medicine 2019). In diesen wird meist zunächst zum Ansprechen der Patienten, zu nachfolgenden Erfassungen des Aktivitätsniveaus sowie der individuellen Patientenperspektive und zur 


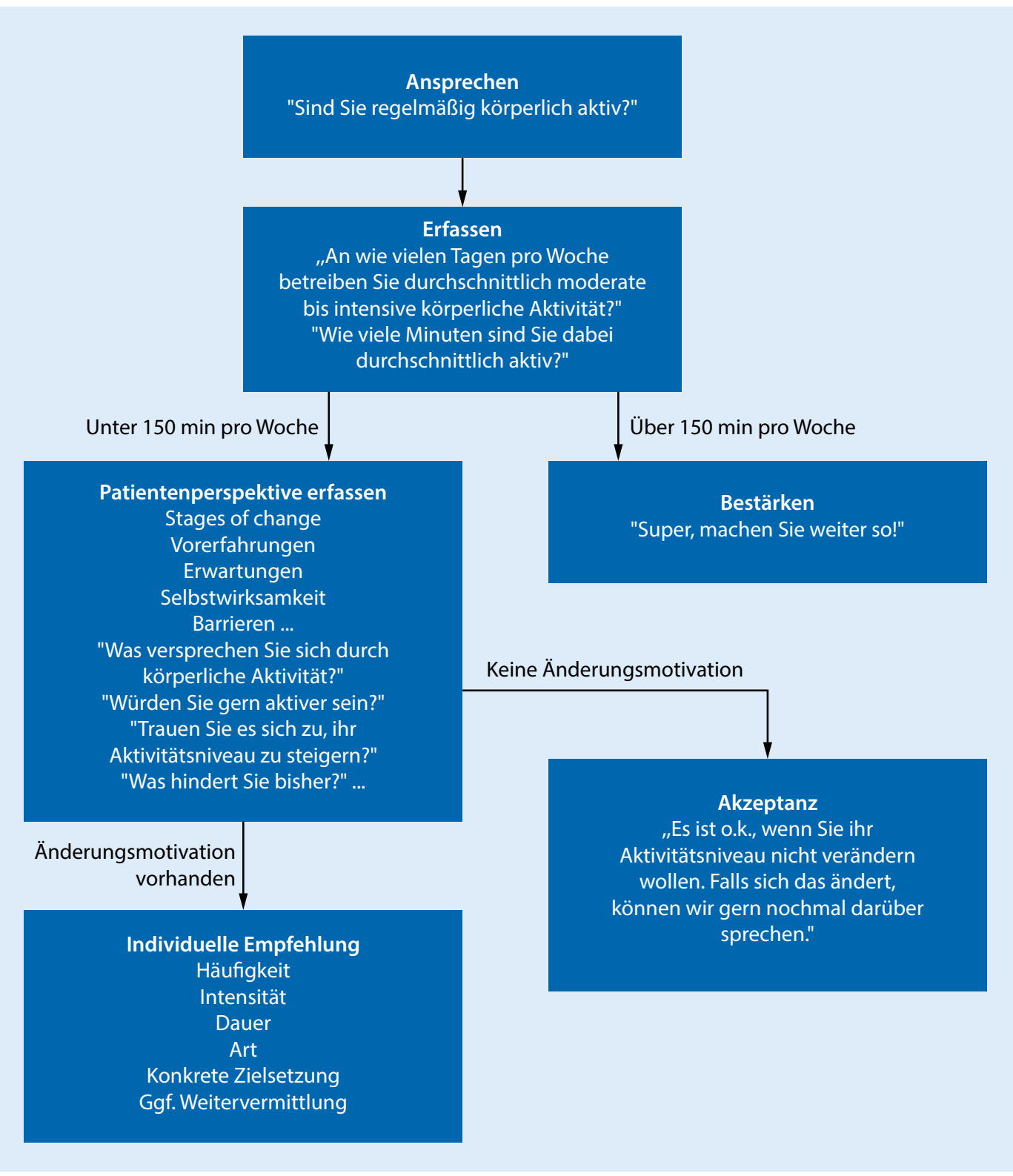

Abb. $1<$ SchemazurEmpfehlung körperlicher Aktivität (Modifiziert nach: Haseler et al. 2019; American College of Sports Medicine 2019)
Entwicklung einer individuellen Aktivitätsempfehlung geraten (• Abb. 1).

Empfehlungen und Ermutigungen hinsichtlich körperlicher Aktivität durch andere Menschen können dabei helfen, das eigene Aktivitätsniveau zu steigern. Zum Beispiel konnte in einer Studie von Bull und Jamrozik (1998) gezeigt werden, dass die ärztliche Beratung bezüglich körperlicher Aktivität inaktiven Patienten helfen kann, ihr Aktivitätsniveau zu steigern. In diesem Zusammenhang spielt die Vorbildfunktion der Behandler eine wichtige Rolle (Lobelo und Quevedo 2016). In einer Studie von Hash et al. (2003) wurde deutlich, dass
Patienten dem Rat ihrer Ärzte mehr vertrauen, wenn diese das empfohlene Gesundheitsverhalten selbst ausüben. Zudem zeigte sich, dass Ärzte, denen ihr eigenes Gesundheitsverhalten wichtig ist, ihren Patienten mehr Empfehlungen zum Gesundheitsverhalten, wie z. B. zur körperliche Aktivität, erteilen (Binns et al. 2007).

Aufgrund der guten Wirksamkeit körperlicher Aktivität im Bereich der psychischen Gesundheit scheint es wichtig, dass Psychiater/ärztliche Psychotherapeuten ihre Patienten über die Vorteile körperlicher Aktivität aufklären. Eine aktuelle Übersichtsarbeit belegt, dass bei Ge- sundheitsfachkräften, die mit psychisch erkrankten Menschen arbeiten, zahlreiche Barrieren bezüglich der Empfehlung körperlicher Aktivität existieren (Glowacki et al.2018). Als eines der häufigsten Hindernisse wurde die unzureichende Ausbildung der Gesundheitsfachkräfte hinsichtlich der Empfehlung körperlicher Aktivität identifiziert. Des Weiteren war ein Großteil der Gesundheitsfachkräfte überzeugt, dass die Betroffenen es nicht schaffen würden, die bei der Aufnahme körperlicher Aktivität bestehenden Schwierigkeiten zu überwinden. Die meisten Studien in diesem Bereich konzentrierten sich auf Pflegekräfte, die 
Psychotherapeut 2020 65:167-175 https://doi.org/10.1007/s00278-020-00421-1

(c) Der/die Autor(en) 2020

M. B. Petzold · F. Ernst · U. Spitzer · J. Gabrysch · N. Schweinfurth · N. Satorius · A. Ströhle · F. Betzler

\section{Ärztliche Vorbildfunktion bezüglich körperlicher Aktivität für Patienten mit psychischen Erkrankungen. Empfehlungsverhalten und Selbstkonkordanz von Psychiatern und ärztlichen Psychotherapeuten}

\section{Zusammenfassung}

Hintergrund. Körperliche Aktivität spielt eine bedeutende Rolle bei der Behandlung psychischer Störungen. Psychiatern/ärztlichen Psychotherapeuten kommt eine Schlüsselrolle dabei zu, ihren Patienten körperliche Aktivität zu empfehlen. Zum Empfehlungsverhalten dieses Kollektivs liegen bisher keine Untersuchungen vor.

Ziele der Arbeit. Das Empfehlungsverhalten in Bezug auf körperliche Aktivität sowie verhaltensbezogene und motivationale Korrelate, die das Empfehlungsverhalten potenziell beeinflussen, bei deutschen Psychiatern/ärztlichen Psychotherapeuten sollte untersucht werden.

Material und Methoden. Insgesamt beantworteten 151 deutsche Psychiater/ärztliche Psychotherapeuten (Alter: $\mathrm{M}=32,5$ Jahre; Geschlecht: 47,0\% weiblich) einen Onlinefragebogen bezüglich körperlicher Aktivität.
Dabei wurden das Empfehlungsverhalten körperlicher Aktivität, die Einstellung, das eigene Aktivitätsverhalten und die Selbstkonkordanz eigener Motivation, körperlich aktiv zu sein, erfasst.

Ergebnisse. Die meisten Psychiater/ärztliche Psychotherapeuten gaben an, körperliche Aktivität einigen $(54,5 \%, n=82)$ oder all ihren Patienten zu empfehlen $(33,8 \%, n=51)$. Das Empfehlungsverhalten korrelierte signifikant positiv mit der Selbstkonkordanz der Motivation $(r=0,19)$ sowie mit der Einstellung zur Bedeutsamkeit körperlicher Aktivität $(r=0,16)$. Hingegen bestand keine signifikante Korrelation zwischen dem eignen Aktivitätsniveau und dem Empfehlungsverhalten der Psychiater/ärztlichen Psychotherapeuten $(r=0,04)$.

Schlussfolgerung. Ein Großteil der Psychiater/ärztlichen Psychotherapeuten empfiehlt körperliche Aktivität. Positiv auf das Empfehlungsverhalten wirkten sich insbesondere die Einschätzung der körperlichen Aktivität als bedeutsam sowie eine hohe Selbstkonkordanz der eigenen Motivation, körperlich aktiv zu sein, aus. Interventionsprogramme, die das Empfehlungsverhalten von Psychiatern/ärztlichen Psychotherapeuten steigern wollen, sollten auf die Selbstkonkordanz der Motivation der Psychiater/ärztlichen Psychotherapeuten, körperlich aktiv zu sein, fokussieren und sich nicht nur auf die Bedeutsamkeit körperlicher Aktivität konzentrieren.

\section{Schlüsselwörter}

Sport · Gesundheitsverhalten · Motivation Deutschland · Umfragen und Fragebogen

\section{Medical exemplary function with respect to physical activity for patients with mental disorders. Recommendation behavior and self-concordance of psychiatrists and medical psychotherapists}

\section{Abstract}

Background. Physical activity (PA) plays an important role in the treatment of mental disorders. Psychiatrists and medical psychotherapists play a key role in this context by recommending PA to their patients. There are no previous studies on the recommendation behavior of psychiatrists for PA.

Objective. The aim of this study was to assess the PA recommendation behavior as well as behavioral and motivational correlates that potentially influence the recommendation behavior of psychiatrists and medical psychotherapists in Germany.

Material and methods. A total of 151 German psychiatrists and medical psychotherapists (age: mean $=32.5$ years; sex: $47.0 \%$ female) answered an online questionnaire on PA assessing recommendation behavior, attitudes, own PA behavior and self-concordance of the motivation to be physically active.

Results. Most psychiatrists and medical psychotherapists recommend PA to some $(54.5 \%, n=82)$ or all of their patients $(33.8 \%$, $n=51)$. Recommendation behavior was significantly positively correlated with selfconcordance of the motivation to perform PA $(r=0.19)$ as well as the attitude with respect to the personal importance of PA $(r=0.16)$. In contrast, there was no significant correlation between the personal activity level and the recommendation behavior of the psychiatrists and medical psychotherapists $(r=0.04)$.
Conclusion. The results show that a large proportion of psychiatrists and medical psychotherapists recommend PA. Attitudes on the importance of PA as well as selfconcordance of the motivation to perform PA showed positive correlations with the recommendation behavior. Intervention programs that aim to improve the recommendation behavior of psychiatrists and medical psychotherapists should focus on the selfconcordance of the psychiatrists and not only on the importance of PA.

\section{Keywords}

Exercise - Health behavior - Motivation . Germany $\cdot$ Surveys and questionnaires mit psychischen erkrankten Menschen arbeiten, und nur eine geringe Zahl von Studien untersuchte auch Psychiater/ ärztliche Psychotherapeuten. Keine der Studien fokussierte explizit die letztere Gruppe (Glowacki et al. 2018). Bisherige Studienergebnisse verdeutlichen, dass neben anderen Faktoren insbesondere das eigene Aktivitätsverhalten der Behandler mit deren Empfehlungsverhalten assoziiert ist (Behandler, die aktiver sind, empfehlen häufiger körperliche Aktivität; Glowacki et al. 2018). Unabhängig von psychischen Erkrankungen wurde sich zudem festgestellt, dass die Selbstkonkordanz der Motivation, körperlich aktiv zu sein, eine wichtige Rolle bei der Aufnahme und
Aufrechterhaltung körperlicher Aktivität spielt (Smith et al. 2007). Die Theorie der Selbstkonkordanz beschreibt zahlreiche Motive für menschliches Handeln, die auf einem Kontinuum der relativen $\mathrm{Au}$ tonomie angeordnet werden (Ryan und Deci 2008). Die Selbstkonkordanz der Motivation drückt aus, inwiefern eine Intention die persönlichen Interessen 


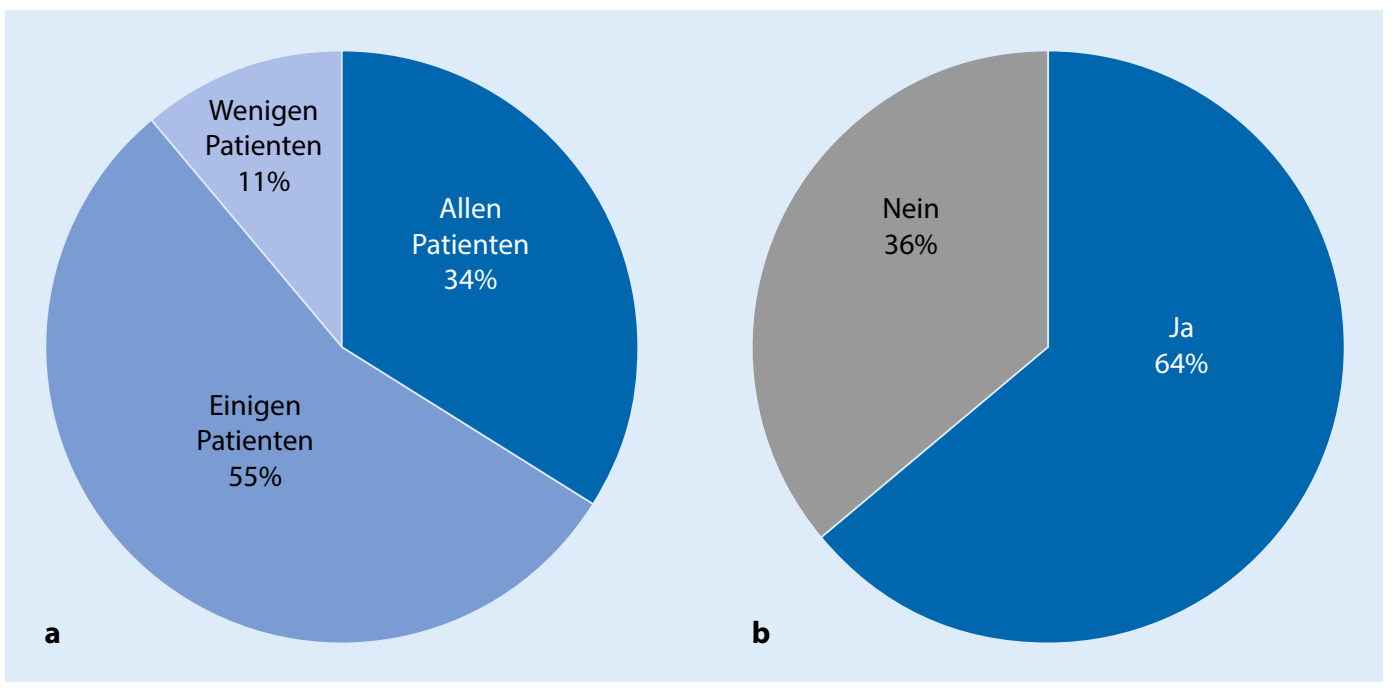

Abb. $2<$ Empfehlungsverhalten (a) und eigenes Aktivitätsniveau (b) der Psychiater/ärztlichen Psychotherapeuten und Werte eines Menschen widerspiegelt (Seelig und Fuchs 2006). Es werden folgende Formen unterschieden: intrinsische („Ich mache Sport, weil es mir Spaß macht"), identifizierte (,... weil es mir gut tut"), extrinsische (,$\ldots$ weil ich sonst Probleme mit anderen Leuten bekomme“) und introjizierte („... weil ich dann ein schlechtes Gewissen habe") Selbstkonkordanz der Motivation (Seelig und Fuchs 2006). Menschen, die intrinsisch motiviert sind, schaffen es mit höherer Wahrscheinlichkeit, ihr Niveau körperlicher Aktivität zu halten, als Menschen, die extrinsisch motiviert sind (Seelig und Fuchs 2006). Aus dem Konzept der Selbstkonkordanz können zudem wichtige Ansatzpunkte für die Beratung von Menschen hinsichtlich ihrer körperlichen Aktivität abgeleitet werden. So postuliert die Theorie die grundlegenden menschlichen Bedürfnisse der Autonomie, Kompetenz und Verbundenheit, die bei der Gestaltung psychologischer Interventionen berücksichtigt werden sollten, und beschreibt, wie ein autonomiefördernder Motivationsstil sich positiv auf die Effekte von Gesundheitsinterventionen auswirken kann (Ryan und Deci 2008). Gemäß dem Wissen der Autoren des vorliegenden Beitrags wurde der Einfluss der Selbstkonkordanz auf das Empfehlungsverhalten körperlicher Aktivität bisher nicht wissenschaftlich untersucht.

\section{Ziel der Arbeit}

Das Empfehlungsverhalten bezüglich körperlicher Aktivität von Psychiatern/ärztlichen Psychotherapeuten in Deutschland sowie der Einfluss des eignen Aktivitätsverhaltens und der Selbstkonkordanz der Motivation, körperlich aktiv zu sein, sollten untersucht werden. Die Hypothese war, dass sowohl das eigene Aktivitätsverhalten als auch die Selbstkonkordanz positiv mit dem Empfehlungsverhalten korrelieren.

\section{Material und Methode}

\section{Stichprobe und Durchführung}

Alle Lehrstuhlinhaber für Psychiatrie in Deutschland wurden per E-Mail angeschrieben und gebeten, ihren Kollegen den Onlinefragebogen über die E-Mail-Verteiler weiterzuleiten. Eine Erinnerung-E-Mail wurde 3 Wochen nach der initialen E-Mail gesendet. Die Teilnehmer wurden gebeten, einen Onlinefragebogen auszufüllen. Sechs Lehrstuhlinhaber antworteten und sendeten den Fragebogen über Verteiler an insgesamt etwa 300 Psychiater/ärztliche Psychotherapeuten. Die Teilnahme war freiwillig, und es erfolgte keine finanzielle Vergütung.

\section{Untersuchungsverfahren}

Der Fragebogen umfasste die Angabe zu soziodemografischen Daten (Alter, Ge- schlecht und Beschäftigungsstatus) der Teilnehmer. Zudem wurden körperliche Aktivität und die subjektive Einschätzung der Bedeutsamkeit körperlicher Aktivität mithilfe von Fragen aus dem Global Physical Activity Questionnaire (Bull et al. 2009) untersucht. Die subjektive Bedeutsamkeit wurde mit der Frage „Wie wichtig ist körperliche Aktivität für Sie in Ihrem Alltag?" gemessen. Die möglichen Antworten reichten von 1 (nicht wichtig) bis 4 (sehr wichtig). Das Aktivitätsniveau wurde erfasst, indem nach dem Ausmaß körperlicher Aktivität pro Woche gefragt wurde. Beispielsweise lauteten die Fragen: „An wie vielen Tagen betreiben Sie moderat-intensiv Sport, Fitness oder Freizeitaktivitäten?" und „Wie viel Zeit verbringen Sie an einem typischen Tag mit moderat-intensivem Sport, Fitness oder Freizeitaktivitäten?“ Um die Intensität verschiedener Aktivitätsarten $\mathrm{zu}$ vergleichen, wurde das metabolische Äquivalent der Aktivitäten („metabolic equivalent of task“, MET) in Anlehnung an frühere Studien berechnet (Garber et al. 2011). Die Selbstkonkordanz der Motivation, körperlich aktiv zu sein, wurde mithilfe der Skala zur Messung der Sport- und Bewegungsbezogenen Selbstkonkordanz (Seelig und Fuchs 2006) erfasst. Zudem wurde das Empfehlungsverhalten der Psychiater/ärztlichen Psychotherapeuten mit der Frage: „Wie oft empfehlen Sie Ihren Patienten körperliche Aktivität?“ ermittelt. Mögliche Antworten auf einer Skala reichten von 1 („keinem 


\begin{tabular}{|c|c|c|c|c|c|c|c|c|}
\hline Variable & 1 & 2 & 3 & 4 & 5 & 6 & 7 & 8 \\
\hline 1. Empfehlungsverhalten & - & - & - & - & - & - & - & - \\
\hline $\begin{array}{l}\text { 2. Subjektive Bedeutsamkeit körperlicher } \\
\text { Aktivität }\end{array}$ & $0,16^{*}$ & - & - & - & - & - & - & - \\
\hline 3. MVPA (min/Woche) & 0,04 & $0,44^{*}$ & - & - & - & - & - & - \\
\hline 4. MET/Woche & 0,11 & $0,50^{* *}$ & $0,96^{* *}$ & - & - & - & - & - \\
\hline 5. SSK-Index & $0,19 *$ & $0,50^{* *}$ & $0,45^{* *}$ & $0,49^{* *}$ & - & - & - & - \\
\hline 6. Intrinsische Motivation & $0,20^{*}$ & $0,66^{* *}$ & $0,48^{* *}$ & $0,52^{* *}$ & $0,79 * *$ & - & - & - \\
\hline 7. Identifizierte Motivation & $0,26^{* *}$ & $0,37^{* *}$ & $0,23^{* *}$ & $0,23^{* *}$ & $0,60^{* *}$ & $0,49^{* *}$ & - & - \\
\hline 8. Introjizierte Motivation & 0,07 & $0,14^{* *}$ & $-0,16$ & $-0,05$ & $-0,36^{* *}$ & 0,08 & $0,19^{*}$ & - \\
\hline 9. Extrinsische Motivation & 0,06 & $-0,06$ & $-0,18^{*}$ & $-0,19^{*}$ & $-0,49^{* *}$ & $-0,15$ & $-0,05$ & $0,22^{*}$ \\
\hline
\end{tabular}

meiner Patienten“) bis 4 („allen meinen Patienten").

\section{Datenanalyse}

Der Anteil fehlender Werte variierte zwischen 0 und 3,3\%, wobei der Mittelwert $2,0 \%$ betrug. Die Ersetzung fehlender Werte erfolgte mithilfe der „multiple imputation“. Diese wurde auf Itembasis durchgeführt. Alle Analysen wurden mit SPSS Statistics 24 anhand der imputierten Datensätze $(n=151)$ berechnet. Die berichteten Ergebnisse sind gemittelte Werte eines Sets von 5 Imputationen. Deskriptive Statistiken wurden zur Analyse der sozidemografischen Variablen und der Analyse des Aktivitätsniveaus der Teilnehmer angewendet. Um Zusammenhänge zwischen den erhobenen Variablen herzustellen, wurden Pearsonund Spearman-Korrelationen berechnet. Das a-Level wurde auf 0,05 festgesetzt (zweiseitig). Aufgrund des eher explorativ-orientierenden Charakters der Studie wurde keine Bonferroni-Korrektur der Signifikanzwerte vorgenommen.

\section{Ergebnisse}

\section{Stichprobe}

Insgesamt beantworteten 151 Personen den Fragebogen. Die Antwortrate betrug damit etwa $50 \%$. Das durchschnittliche Alter der Teilnehmer betrug 32,5 Jahre (Standardabweichung [SD] $\pm 5,6$ Jahre). Von den Teilnehmern waren $47,0 \%$ $(n=71)$ weiblich, $52,4 \%(n=79)$ männ- lich, und 0,7\% $(n=1)$ machten keine Angaben hinsichtlich des Geschlechts. Es waren $53,0 \% \quad(n=80) \quad$ Assistenzärzte/in Psychotherapieweiterbildung, $33,1 \%(n=50)$ waren Fachärzte für Psychiatrie/ärztliche Psychotherapeuten, $8,6 \%(n=13)$ waren Klinikdirektoren, und 5,3\% $(n=8)$ teilten mit, in einer anderen Position $\mathrm{zu}$ arbeiten.

\section{Deskriptive Ergebnisse}

Die meisten Teilnehmer gaben an, dass körperliche Aktivität ihnen wichtig (48,3\%, n=73) oder sehr wichtig $16,6 \%$ $(n=19)$ ist. Etwa ein Drittel der Teilnehmer hielt körperliche Aktivität für weniger wichtig $(33,1 \%, n=50)$ oder nicht wichtig $(5,3 \%, n=8)$. Die Mehrheit der Psychiater/ärztlichen Psychotherapeuten erklärte, körperliche Aktivität einigen ihrer Patienten $\mathrm{zu}$ empfehlen $(54,3 \%, n=82)$, etwa ein Drittel sogar allen Patienten (33,8\%, $n=51)$. Körperliche Aktivität nur wenigen Patienten zu empfehlen, gaben $11,3 \%(n=17)$ an; keiner der Teilnehmer berichtete, körperliche Aktivität keinem seiner Patienten zu empfehlen (• Abb. 2).

Die Teilnehmer erklärten, durchschnittlich 223,2 min moderate bis intensive körperliche Aktivität („moderate-tovigorous physical activity“, MVPA) pro Woche zu betreiben ( $S D \pm 96,5 \mathrm{~min})$. Der Mittelwert der intensiven Aktivität pro Woche betrug 79,9 $\min (S D \pm 96,5 \mathrm{~min})$, der Mittelwert der moderaten Aktivität pro Woche 143,31 $\mathrm{min}$ ( $S D \pm 129,9 \mathrm{~min})$. Dies entspricht einem mittleren Ver- brauch von 1212,0 MET/Woche. Etwa zwei Drittel der Teilnehmer (63,6\%, $n=96)$ erreichten die Empfehlung der Weltgesundheitsorganisation und betrieben mindestens $150 \mathrm{~min}$ moderate bis intensive körperlicher Aktivität/Woche (• Abb. 1). Etwa die Hälfte der Teilnehmer war mindestens 5-mal/Woche 30 min moderat bis intensiv körperlich aktiv $(47,7 \%, n=72)$.

\section{Zusammenhänge mit dem Empfehlungsverhalten}

Die Interkorrelationen zwischen Empfehlungsverhalten, körperlicher Aktivität, subjektiver Bedeutsamkeit körperlicher Aktivität und Selbstkonkordanz sind in - Tab. 1 aufgeführt.

Es fanden sich signifikante positive Korrelationen zwischen dem Empfehlungsverhalten, der subjektiven Bedeutsamkeit körperlicher Aktivität und der Selbstkonkordanz, nicht jedoch mit dem Ausmaß körperlicher Aktivität, das die Psychiater/ärztlichen Psychotherapeuten selbst betrieben. Bezüglich der einzelnen Subskalen der Selbstkonkordanz ergaben sich positive Korrelationen der intrinsischen und identifizierten, nicht jedoch introjizierten oder extrinsischem Motivation mit dem Empfehlungsverhalten. Zudem zeigten sich signifikante Korrelationen zwischen der subjektiven Bedeutsamkeit körperlicher Aktivität und dem eigenen Aktivitätsverhalten sowie der Selbstkonkordanz der Motivation. Neben dem Empfehlungsverhalten und der subjektiven Bedeutsamkeit körperlicher 
Aktivität war auch die Selbstkonkordanz positiv mit dem Aktivitätsniveau korreliert.

\section{Diskussion}

\section{Interpretation der Studienergebnisse}

Die vorgestellten Ergebnisse zeigen, dass die Mehrheit der Psychiater/ärztlichen Psychotherapeuten angibt, einigen ihrer Patienten körperliche Aktivität zu empfehlen. Ein gutes Drittel empfiehlt sie sogar allen Patienten. Nur eine kleine Gruppe von Psychiatern/ärztlichen Psychotherapeuten teilt mit, nur wenigen Patienten körperliche Aktivitäten zu empfehlen. In Anbetracht der zahlreichen positiven Effekte auf die psychische Gesundheit und bezogen auf die Tatsache, dass niemand angab, keine körperliche Aktivität zu empfehlen, ist dieses Ergebnis ermutigend. Dennoch existiert eine Gruppe von Psychiatern/ ärztlichen Psychotherapeuten, die nur wenigen Patienten körperliche Aktivität empfiehlt. Vor dem Hintergrund der wachsenden Evidenz dafür, dass körperliche Aktivität bei der Behandlung von nahezu allen psychischen Erkrankungen hilfreich sein kann (Ströhle 2019), ist die Empfehlung zu körperlicher Aktivität bei einem sehr großen Teil der Patienten wertvoll. Die Psychiater/ärztliche Psychotherapeuten betreiben insgesamt ein ähnliches Ausmaß an körperlicher Aktivität wie die Allgemeinbevölkerung (Bertheussen et al. 2011). Bezüglich des Empfehlungsverhaltens wurde keine Korrelation mit dem eigenen Aktivitätsniveau festgestellt. Dieses Ergebnis weicht von den Erkenntnissen früherer Studien ab (Glowacki et al. 2018) und widerspricht der oben genannten Hypothese. Eine mögliche Erklärung für die Zusammenhänge zwischen körperlicher Aktivität und dem Empfehlungsverhalten in früheren Studien könnte der Einfluss der Motivation auf beide dieser Faktoren sein, sodass dieser gemeinsame Einflussfaktor zu einer Korrelation der Variablen führt. Zur detaillierteren Untersuchung der Zusammenhänge zwischen diesen Variablen scheinen weitere Studien notwendig, wobei auch eine ausführlichere Erfassung körperlicher Aktivität empfohlen wird. Die Vorbildfunktion von Ärzten wurde zuvor in 2 Studien (Frank et al. 2013 und Hash et al. 2003) untersucht. Beide Studien kamen zu dem Ergebnis, dass Ärzte umso häufiger körperliche Aktivität empfehlen, je mehr sie selbst körperlich aktiv sind. Eine weitere Studie von Stanton et al. (2015) konnte jedoch, ebenso wie die vorliegende, keinen Zusammenhang feststellen. Im Kontrast zu der Studie von Hash et al. (2003) untersuchten die vorliegende Studie und die Studie von Stanton et al. (2015) keine Zusammenhänge mit der Akzeptanz der Empfehlungen durch die Patienten. Zudem fand keine Prüfung, inwiefern die Patienten den Empfehlungen tatsächlich Folge leisten, statt. Eine ausführlichere Erfassung der Perspektive der Patienten und der Adhärenz bezüglich der Empfehlungen könnte zu einer differenzierteren Darstellung und einem tieferen Verständnis der Thematik führen.

Hingegen wurde in der vorliegenden Studie eine positive Korrelation zwischen intrinsischer Motivation, körperlich aktiv zu sein, und dem Empfehlungsverhalten gefunden, was der oben genannten Hypothese entsprach. Dieses Ergebnis kann darauf hindeuten, dass die Selbstkonkordanz der Motivation, körperlich aktiv zu sein, eine größere Rolle spielt als das Ausmaß der selbst betriebenen körperlichen Aktivität. Für ein weitergehendes Verständnis der Zusammenhänge sind jedoch weitere Studien, insbesondere in Form einer differenzierteren Erfassung des Empfehlungsverhaltens in Kombination mit dem eigenen Aktivitätsniveau und der Selbstkonkordanz notwendig, bei denen das Zusammenspiel dieser Variablen beispielsweise mithilfe von Pfadanalysen untersucht wird.

Teilnehmer, die zwar häufig körperlich aktiv, gleichzeitig jedoch extrinsisch motiviert waren, empfahlen weniger häufig körperliche Aktivität als Teilnehmer, die zwar weniger körperlich aktiv, dafür jedoch intrinsisch motiviert waren. Basierend auf diesen Ergebnissen sollten zukünftige Studien die Rolle der Selbstkonkordanz bezüglich körperlicher Aktivität als wichtigen Ein- flussfaktor weiteruntersuchen, und die Art der Motivation sollte als wichtiger vermittelnder Faktor bzw. Barriere betrachtet werden. Eine Schlussfolgerung aus den vorliegenden Ergebnissen könnte daher sein, dass Interventionen, die das Empfehlungsverhalten körperlicher Aktivität bei Psychiatern/ärztlichen Psychotherapeuten fördern sollen, sich insbesondere auf die Selbstkonkordanz der Motivation, körperlich aktiv zu sein, konzentrieren sollten. Diesem Konzept folgend könnten Psychiatern/ärztlichen Psychotherapeuten verschiedene Arten körperlicher Aktivität nähergebracht werden. Der Ansatz, den Fokus dabei auf Spiel, Spaß und Wohlbefinden zu legen (was die intrinsische Motivation fördert), könnte sich als vielversprechender erweisen als die reine Information über die potenziellen Vorteile körperlicher Aktivität (was die extrinsische Motivation verstärken könnte). Gleichzeitig handelt es sich bei der Selbstkonkordanz der Motivation um ein Kontinuum, wobei als Startpunkt zunächst extrinsische Motivationen hilfreich sein können, um in Aktion zu kommen, woraufhin über Prozesse u. a. der Introjektion die Selbstkonkordanz der Motivation erhöht werden kann. Wie erwartet, bestehen positive Korrelationen zwischen der Selbstkonkordanz der Motivation, körperlich aktiv zu sein, und dem Aktivitätsniveau sowie der subjektiven Bewertung der Bedeutsamkeit körperlicher Aktivität. Vorangegangene Studien konnten zeigen, dass eine intrinsische Motivation der wichtigste Faktor ist, um körperliche Aktivität aufrechtzuerhalten (Lippke und Vögele 2006). Intrinsisch motiviert zu sein, könnte dazu führen, dass körperlicher Aktivität eine größere subjektive Bedeutsamkeit beigemessen und mehr körperliche Aktivität betrieben wird. Diese Ergebnisse stehen im Einklang mit den Erkenntnissen von Koestner et al. (2002), dass Menschen, die ein Ziel aufgrund ihrer persönlichen Interessen erreichen wollen, eher erfolgreich sind. Außer den in dieser Untersuchung fokussierten motivationalen Faktoren, die insbesondere bei der Intentionsbildung bedeutsam sind, spielen auch volitionale Faktoren hinsichtlich der Aufnahme und Aufrechterhaltung kör- 
perlicher Aktivität eine wichtige Rolle. Diese fokussieren Prozesse der Selbstregulation und Selbststeuerung, die bei der Umsetzung der gebildeten Intentionen in tatsächliche Handlungen relevant sind (Fuchs 2013). Abgesehen von der reinen Empfehlung scheinen aus dieser Perspektive insbesondere die durch Psychiater/ärztliche Psychotherapeuten idealerweise zu vermittelnden Strategien der konkreten Handlungsplanung und des Barrieremanagements von Interesse. Eine Erfassung der Vermittlung derartiger Strategien wird daher für zukünftige Studien dringend empfohlen.

\section{Stärken der Studie}

Die vorliegende Studie ist nach Wissen der Autoren die erste Studie, die das Empfehlungsverhalten körperlicher Aktivität bei Psychiatern/ärztlichen Psychotherapeuten untersucht und die weltweit erste Studie, die Zusammenhänge mit der Selbstkonkordanz herstellt. Die Stichprobe aus 151 Psychiatern/ärztlichen Psychotherapeuten ist begrenzt, im Vergleich $\mathrm{zu}$ vorherigen Studien jedoch relativ groß. Zudem ist die Zielpopulation klarer spezifiziert als in Studien, in denen zahlreiche unterschiedliche Berufsgruppen gemeinsam untersucht wurden (Glowacki et al. 2018).

\section{Limitationen der Studie}

Dennoch sind folgende wichtige Limitationen $\mathrm{zu}$ beachten. Erstens ist es möglich, dass Psychiater/ärztliche Psychotherapeuten, die körperlich aktiver oder an dem Thema besonders interessiert sind, mit höherer Wahrscheinlichkeit gewillt waren, den Fragebogen auszufüllen. Dies könnte zu einem Stichprobenfehler mit einer Überschätzung des Empfehlungsverhaltens geführt haben. Bezüglich der Repräsentativität der Stichprobe wurden die Alters- und die Geschlechtsverteilung mit den Daten der Bundesärztekammer verglichen. In der Stichprobe der vorliegenden Untersuchung lag eine $\mathrm{Ge}$ schlechtsverteilung vor (47,00\% Frauen; $52,70 \%$ Männer), die sich leicht von der aller Psychiater/ärztlichen Psychotherapeuten in Deutschland (53,53\% Frauen; 46,47\% Männer) unterschied. Der größte Anteil der Teilnehmer gab an, unter 34 Jahre alt zu sein, wohingegen der größte Anteil der Psychiater/ärztlichen Psychotherapeuten der offiziellen Statistik nach zwischen 50 und 59 Jahren alt ist. Das jüngere Alter der an der vorliegenden Studie Teilnehmenden könnte durch die Verwendung des Onlinefragebogens erklärt werden. Jüngere Generationen könnten häufiger auf MailingListen vertreten sein und eine höhere Affinität zu Onlinediensten haben, sodass ältere, insbesondere niedergelassene Psychiater/ärztliche Psychotherapeuten nur unzureichend erreicht worden sein könnten. Daher könnte die Repräsentativität dieser Studie auf eher junge Psychiater/ärztliche Psychotherapeuten in stationären Settings beschränkt sein.

Zudem wurde das Empfehlungsverhalten lediglich mithilfe einer einzigen Frage erfasst, um den Fragebogen kurz zu halten. So wurde das genaue Ausmaß, das die Psychiater/ärztlichen Psychotherapeuten empfehlen, z. B. hinsichtlich der Häufigkeit, der Intensität und der Art körperlicher Aktivität, nicht ermittelt. Auch bezüglich des Anteils der Patienten, denen die Psychiater/ärztlichen Psychotherapeuten körperliche Aktivität empfehlen, fand lediglich eine grobe Differenzierung statt („keine“, „wenige“, „einige“, „alle“). Für zukünftige Studien wäre diesbezüglich eine differenziertere Einschätzung, z. B. über Prozentwerte, wünschenswert. Eine ausführlichere Untersuchung des Empfehlungsverhaltens, beispielsweise mithilfe des zwischenzeitlich entwickelten „Exercise in Mental Illness Questionnaire“ (Stanton et al. 2014) wird für zukünftige Studien dringend empfohlen. Eine weitere Limitation stellt die Erfassung lediglich durch Selbstberichte dar. Der Einsatz objektiver Messverfahren, z.B. von Akzelerometern, könnte die Zuverlässigkeit der Ergebnisse steigern. Zudem wurde ein sehr kurzes Messverfahren zur Erfassung körperlicher Aktivität angewendet. Für zukünftige Studien empfiehlt sich die Nutzung ausführlicherer Fragebogen, beispielsweise des neu entwickelten Simple Physical Activity Questionnaire (SIMPAQ) (Rosenbaum et al. 2020). Weiterhin wurde nicht untersucht, in- wiefern die Psychiater/ärztlichen Psychotherapeuten körperliche Aktivität wie angegeben tatsächlich empfahlen, oder ob die Patienten den Empfehlungen Folge leisteten. Rückmeldungen von Patienten einzuholen, könnte diese Limitationen reduzieren. Diese und die zuvor genannten Einschränkungen sollten bei der Interpretation der Ergebnisse berücksichtigt werden.

\section{Ausblick}

Die vorliegende Studie ist die erste wissenschaftliche Untersuchung, die das Empfehlungsverhalten körperlicher Aktivität bei Psychiatern/ärztlichen Psychotherapeuten in Deutschland untersucht. Sie zeigt, dass die Mehrheit der Psychiater/ärztlichen Psychotherapeuten körperliche Aktivität einigen ihrer Patienten empfiehlt, wenngleich dies nur bei knapp einem Drittel für alle Patienten der Fall ist. Die Studie liefert zudem Hinweise, dass die intrinsische Motivation eine wichtige Rolle gleichermaßen für die Empfehlung als auch für das eigene Aktivitätsniveau der Psychiater/ärztlichen Psychotherapeuten, spielt. Intrinsisch zu körperlicher Aktivität motiviert zu sein, führt eher dazu, dass auch Patienten körperliche Aktivität empfohlen wird.

Bei der Entwicklung von Interventionsprogrammen bzw. Weiterbildungsveranstaltungen zur Empfehlung körperlicher Aktivität für Psychiater/ärztliche Psychotherapeuten sollten motivationale Faktoren Berücksichtigung finden. Dabei sollte nicht nur die reine Informationsvermittlung im Vordergrund stehen, sondern auch die Selbstkonkordanz der eigenen Motivation adressiert werden, was neben motivationalen Interventionen durch sportliche Selbsterfahrung denkbar wäre.

\section{Fazit für die Praxis}

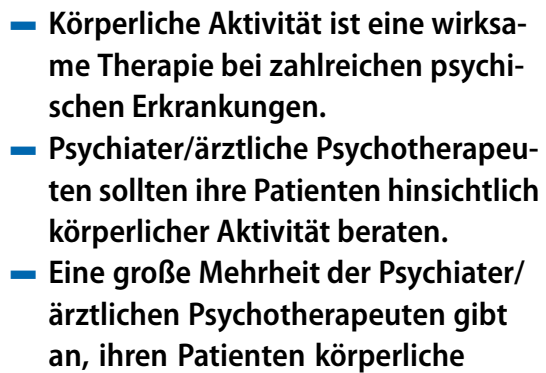

Psychotherapeut $\mathbf{3} 2020$ 


\section{Aktivität zu empfehlen, wenngleich dies lediglich bei etwa einem Drittel für alle Patienten zutrifft. \\ - Psychiater/ärztliche Psychotherapeu- ten, die bezüglich ihrer körperlichen Aktivität intrinsisch motiviert sind, empfehlen diese ggf. eher. \\ - Das eigene Aktivitätsniveau hatte keinen Einfluss auf das Empfehlungs- verhalten.}

\section{Korrespondenzadresse}

\section{Dr. rer. medic. Moritz Bruno Petzold}

Klinik für Psychiatrie und Psychotherapie, Charité - Universitätsmedizin Berlin, Campus Mitte, corporate member of Freie Universität Berlin, Humboldt-Universität zu Berlin, and Berlin Institute of Health Charitéplatz 1, 10117 Berlin, Deutschland moritz.petzold@charite.de

Funding. Open Access funding provided by Projekt DEAL.

\section{Einhaltung ethischer Richtlinien}

Interessenkonflikt. M.B. Petzold, F. Ernst, U. Spitzer, J. Gabrysch, N. Schweinfurth, N. Satorius, A. Ströhle und F. Betzler geben an, dass kein Interessenkonflikt besteht.

Alle beschriebenen Untersuchungen wurden im Einklang mit nationalem Recht sowie gemäß der Deklaration von Helsinki von 1975 (in der aktuellen, überarbeiteten Fassung) durchgeführt. Die Umfrage erfolgte anonym, es wurden keine Personenbezogenen Daten erhoben. Eine gremiale Prüfung durch die Ethikkommission entfiel. Alle Teilnehmerinnen und Teilnehmer gaben vor Teilnahme ihre Einverständniserklärung.

Open Access. Dieser Artikel wird unter der Creative Commons Namensnennung 4.0 International Lizenz veröffentlicht, welche die Nutzung, Vervielfältigung, Bearbeitung, Verbreitung und Wiedergabe in jeglichem Medium und Format erlaubt, sofern Sie den/die ursprünglichen Autor(en) und die Quelle ordnungsgemäß nennen, einen Link zur Creative Commons Lizenz beifügen und angeben, ob Änderungen vorgenommen wurden.

Die in diesem Artikel enthaltenen Bilder und sonstiges Drittmaterial unterliegen ebenfalls der genannten Creative Commons Lizenz, sofern sich aus der Abbildungslegende nichts anderes ergibt. Sofern das betreffende Material nicht unter der genannten Creative Commons Lizenz steht und die betreffende Handlung nicht nach gesetzlichen Vorschriften erlaubt ist, ist für die oben aufgeführten Weiterverwendungen des $\mathrm{Ma}$ terials die Einwilligung des jeweiligen Rechteinhabers einzuholen.

Weitere Details zur Lizenz entnehmen Sie bitte der Lizenzinformation auf http://creativecommons.org/ licenses/by/4.0/deed.de.

\section{Literatur}

American College of Sports Medicine (2019) Health care providers action guide. https://www.exerciseismedicine.org/assets/ page_documents/EIM\%20Health\%20Care $\% 20$ Providers\%20Action\%20Guide\%20clickable \%20links.pdf.Zugegriffen:23.Jan. 2020

Ashdown-Franks G, Sabiston CM, Stubbs B (2019) The evidence for physical activity in the management of major mental illnesses: a concise overview to inform busy clinicians' practice and guide policy. Curr Opin Psychiatry 32(5):375-380. https://doi. org/10.1097/YCO.0000000000000526

Bertheussen GF, Romundstad PR, Landmark T, Kaasa S, Dale O, Helbostad JL (2011) Associations between physical activity and physical and mental health- A HUNT 3 study. Med Sci Sports Exerc 43(7):1220-1228. https://doi.org/ 10.1249/MSS.0b013e318206c66e

Binns HJ, Mueller MM, Ariza AJ (2007) Healthy and fit for prevention: the influence of clinician health and fitness on promotion of healthy lifestyles during health supervision visits. Clin Pediatr (Phila) 46:780-786. https://doi.org/10.1177/ 0009922807303229

Bull FC, Jamrozik K (1998) Advice on exercise from a family physician can help sedentary patients to become active. Am J Prev Med 15:85-94. https:// doi.org/10.1016/50749-3797(98)00040-3

Bull FC, Maslin TS, Armstrong T (2009) Global physical activity questionnaire (GPAQ): nine country reliability and validity study. J Phys Act Health 6(6):790-804. https://doi.org/10.1123/jpah.6.6. 790

Czosnek L, Lederman O, Cormie P, Zopf E, Stubbs B, Rosenbaum S (2018) Health benefits, safety and cost of physical activity interventions for mental health conditions: A meta-review to inform translation efforts. Ment Health Phys Act 16:140-151. https://doi.org/10.1016/j.mhpa. 2018.11.001

Frank E, Dresner Y, Shani M, Vinker S (2013) The association between physicians' and patients' preventive health practices. Cmaj 185(8):649-653. https://doi.org/10.1503/cmaj.121028

Fuchs R (2013) Das Motivations-Volitions-Konzept. Public Health Forum 21(2):32-34. https://doi. org/10.1016/j.phf.2013.03.004

Garber CE, Blissmer B, Deschenes MR, Franklin BA, Lamonte MJ, Lee I-M, Nieman DC, Swain DP (2011) Quantity and quality of exercise for developing and maintaining cardiorespiratory, musculoskeletal, and neuromotor fitness in apparently healthy adults. Med Sci Sports Exerc 43:1334-1359. https://doi.org/10.1249/MSS. 0b013e318213fefb

Glowacki K, Weatherson K, Faulkner G (2018) Barriers and facilitators to health care providers' promotion of physical activity for individuals with mental illness: a scoping review. Ment Health Phys Act 16:152-168. https://doi.org/10. 1016/j.mhpa.2018.10.006

Haseler C, Crooke R, Haseler T (2019) Promoting physical activity to patients. BMJ 366:15230. https://doi.org/10.1136/bmj.15230

Hash RB, Munna RK, Vogel RL, Bason JJ (2003) Does physician weight affect perception of health advice? Prev Med 36:41-44. https://doi.org/10. 1006/pmed.2002.1124

Koestner R, Lekes N, Powers TA, Chicoine E (2002) Attaining personal goals: Self-concordance plus implementation intentions equals success.J Pers SocPsychol 83(1):231-244
KruisdijkF, DeenikJ, TenbackD, TakE, Beekman A-J, van Harten P, Hopman-Rock M, Hendriksen I (2017) Accelerometer-measured sedentary behaviour and physical activity of inpatients with severe mental illness. Psychiatry Res 254:67-74. https:// doi.org/10.1016/j.psychres.2017.04.035

Lippke S, Vögele C (2006) Sport und körperliche Aktivität. In: Renneberg B, Hammelstein P (Hrsg) Gesundheitspsychologie. Springer, Berlin Heidelberg New York, S 195-216

Lobelo F, de Quevedo IG (2016) The evidence in support of physicians and health care providers as physical activity role models. Am J Lifestyle Med 10:36-52. https://doi.org/10. 1177/1559827613520120

Petzold MB, BischoffS, Rogoll J, Plag J, Terán C, Brand R, Ströhle A (2017) Physical activity in outpatients with mental disorders: status, measurement and social cognitive determinants of health behavior change. Eur Arch Psychiatry Clin Neurosci 267:639-650. https://doi.org/10.1007/s00406017-0772-3

Petzold MB, Bendau A, Ströhle A (2020) Körperliche Aktivität in der Behandlung von Angsterkrankungen. Psychotherapeut. https://doi.org/10. 1007/s00278-020-00414-0

Rosenbaum S, Ward PB, Baldeo R, Fibbins $\mathrm{H}$, Jarman R, Lederman O, Perram A, Poole J, Rossimel E, Smith G, Teasdale S, Wade T, Watkins A, White A, Pearce D, Curtis J (2020) Changing health workforce attitudes to promote improved physical health in mental health service users: keeping our staff in mind (KosiM). Health Promot J Austral. https://doi.org/10.1002/hpja.320

Ryan RM, Deci EL (2008) A self-determination theory approach to psychotherapy:the motivational basis for effective change. Can Psychol 49(3):186-193. https://doi.org/10.1037/ a0012753

Schuch F, Vancampfort D, Firth J, Rosenbaum S, Ward $P_{\text {, }}$ Reichert T, Bagatini NC, Bgeginski R, Stubbs B (2017) Physical activity and sedentary behavior in people with major depressive disorder: a systematic review and meta-analysis. J Affect Disord 210:139-150. https://doi.org/10.1016/j. jad.2016.10.050

Schuch FB, Vancampfort D, Firth J, Rosenbaum S, Ward $\mathrm{PB}$, Silva $\mathrm{ES}$, Hallgren $M$, Ponce De Leon A, Dunn AL, Deslandes AC, Fleck MP, Carvalho AF, Stubbs B (2018) Physical activity and incident depression: a meta-analysis of prospective cohort studies. Am J Psychiatry 175:631-648. https://doi.org/10.1176/appi.ajp. 2018.17111194

Seelig H, Fuchs R (2006) Messung der sportund bewegungsbezogenen Selbstkonkordanz. Z Sportpsychol 13:121-139. https://doi.org/10. 1026/1612-5010.13.4.121

Smith A, Ntoumanis N, Duda J (2007) Goal striving, goal attainment, and well-being: adapting and testing the self-concordance model in sport. JSportExercPsychol 29:763-782

Stanton R, Happell B, Reaburn P (2014) The development of a questionnaire to investigate the views of health professionals regarding exercise for the treatment of mental illness. Ment Health Phys Act 7:177-182. https://doi.org/10.1016/j.mhpa. 2014.06.001

Stanton R, Reaburn P, Happell B (2015) Barriers to exercise prescription and participation in people with mental illness: the perspectives of nurses working in mental health. J Psychiatr Ment Health Nurs 22:440-448. https://doi.org/10. 1111/jpm.12205 
Ströhle A (2019) Sports psychiatry: mental health and mental disorders in athletes and exercise treatment of mental disorders. Eur Arch PsychiatryClin Neurosci269(5):485-498.https:// doi.org/10.1007/s00406-018-0891-5

Ströhle A, Höfler M, Pfister H, Müller A-G, Hoyer J, Wittchen H-U, Lieb R (2007) Physical activity and prevalence and incidence of mental disorders in adolescents and young adults. Psychol Med 37:1657-1666. https://doi.org/10.1017/ S003329170700089X

Vancampfort D, Rosenbaum S, Probst M, Connaughton J, Du Plessis C, Yamamoto T, Stubbs B (2016) What are the top 10 physical activity research questions in schizophrenia? Disabil Rehabil 38:2235-2243. https://doi.org/10.3109/ 09638288.2015 .1116622

Zschucke E, Gaudlitz K, Ströhle A (2013) Exercise and physical activity in mental disorders: clinical and experimental evidence.J J Prev Med Public Health 46(Suppl 1):S12-S21. https://doi.org/10.3961/ jpmph.2013.46.S.S12

\section{Wir sind „aktiv Handelnde“ \\ Psychologen belegen, dass das menschliche Gehirn künftige Handlungseffekte vorhersagt}

Der Mensch gilt gemeinhin als vorausschauend handelndes Wesen. Sein Gehirn ist in der Lage, künftige Handlungsmuster und deren Konsequenzen in der Umwelt vorherzusagen. Zielgerichtetes Handeln ist damit möglich.

Vorhersagen auf neuronaler Ebene nachgewiesen

In einer Studie hat ein Forschungsteam um

Dr. David Dignath und Prof. Dr. Andrea Kiesel vom Institut für Psychologie der Universität Freiburg sowie Kollegen von der Universität Trier untersucht, wie solche Vorhersagen auf neuronaler Ebene aussehen. „Wir sind davon ausgegangen, dass es eine neuronale Aktivität gibt, welche die Erwartungshaltung widerspiegelt und dem Handeln vorausgeht", erläutert Kiesel. Um das zu testen, zeichneten die Forschenden die Hirnaktivität von insgesamt 60 Probandinnen und Probanden mithilfe eines Elektroenzephalogramms (EEG) auf. „Die Ergebnisse zeigen, dass sich die Erwartung dessen, was wir in der Umwelt verändern, bereits vor Beginn einer Handlung in der Hirnaktivität messen lässt", sagt Dignath. Die Resultate bestätigen damit die Annahme der Wissenschaftlerin und Wissenschaftler und stützen klassische Theorien de Handlungskontrolle. Ihre Ergebnisse präsentieren sie in der Fachzeitschrift "Journal of Experimental Psychology: General“.

\section{Aktiv Handelnde anstatt passiv} Reagierende

Theorien zur psychologischen Handlungskontrolle besagen, dass Menschen handeln um etwas in ihrer Umgebung zu bewirken. Sie erzeugen dadurch absichtlich bestimmte Handlungseffekte - und sind somit aktiv Handelnde und nicht passiv Reagierende.

„Beim Klavierspielen werden die Tasten gedrückt, um entweder nur eine Bewegung zu machen, die durch die Notenanordnung auf dem Notenblatt bestimmt ist, oder aber, um eine Melodie als Effekt zu erzeugen", sagt Kiesel. Ziel der vorliegenden Studie war es nachzuweisen, dass solche Effekte im EEG messbar sind, während eine Handlung geplant, aber noch nicht ausgeführt wird. Während der Experimente drückten die Probanden einige hundert Male auf zwei verschiedene Computertasten. Beim Drücken der einen Taste erschien das Bild, das mit einer Frequenz von sechs Hertz flackerte. Beim Druck auf die andere Taste erschien ein Bild, das um einiges schneller, nämlich mit einer Frequenz von zehn Hertz, flackerte. Durch abwechselndes Drücken der Tasten lernten die Teilnehmenden, wann welches Bild erschien, und entwickelten eine entsprechende Erwartungshaltung. „Das Gehirn reagiert besonders stark auf die Frequenz, in der das jeweilige Bild gezeigt wird, in diesem Experiment also entweder sechs oder zehn Hertz", erläutert Dignath. Das wiederum zeige sich im EEG, während Probanden die flackernden Bilder beobachteten, die auf den Tastendruck folgten.

\section{Antizipation bereits vor der} eigentlichen Handlung sichtbar Neu ist, dass die Forschenden nachweisen konnten, dass sich aus dem EEG-Signal bereits vor Beginn der Handlung auslesen lässt, welches Bild erzeugt wird. So zeigte die Gehirnaktivität im EEG ein charakteristisches Muster, bevor eine Taste gedrückt wird. "Unser Gehirn funktioniert über Kontraste", erklärt Dignath. Das bedeute, dass das Gehirn, kurz bevor der Proband eine der beiden Tasten drücke, jeweils in einem bestimmten Bereich weniger neuronale Aktivität zeige als im Ruhezustand. „Erscheint das Bild dann tatsächlich, fährt die Aktivität in diesem Bereich hoch, sodass es schnell registriert wird. Das Besondere daran ist, dass diese Antizipation direkt im EEG sichtbar ist, noch bevor die eigentliche Handlung ausgeführt wird", betont Dignath.

Originalpublikation: Dignath, D., Kiesel, A., Frings, C., \& Pastötter, B. (2020). Electrophysiological evidence for actioneffect prediction. Journal of Experimental Psychology: General, 149(6), 1148-1155. https://doi.org/10.1037/xge0000707.

Quelle: Albert-Ludwigs-Universität Freiburg im Breisgau (www.pr.unifreiburg.de/pm;07.05.2020) 